\title{
Journal of Open Public Health Data launched
}

Eurosurveillance editorial team (eurosurveillance@ecdc.europa.eu)1

1. European Centre for Disease Prevention and Control (ECDC), Stockholm, Sweden

Citation style for this article:

Eurosurveillance editorial team. Journal of Open Public Health Data launched. Euro Surveill. 2013;18(33):pii=20558. Available online: http://www.eurosurveillance. org/ViewArticle.aspx?Articleld $=20558$

Article published on 15 August 2013

The Journal of Open Public Health Data (JOPHD) was launched in July this year. It aims to incentivise the publication of openly available public health datasets according to community standards [1]. JOPHD publishes data papers, which do not contain research results but rather a description of a dataset, and where to find it. The journal accepts only papers for datasets that authors agree to make freely available in a public repository. This means that they have been deposited in a data repository under an open licence, such as a Creative Commons Zero licence [2], and are therefore freely available to anyone with an Internet connection, anywhere in the world.

A data paper is a publication that is designed to make other researchers aware of data that are of potential use to them for scientific and educational purposes. Data papers can describe deposited data from studies that have not been published elsewhere (including replication research) but also from studies that have previously been published in another journal. As such, the data paper describes the methods used to create the dataset, its structure, its reuse potential, and a link to its location in a repository. It is important to note that a data paper does not replace a research article, but complements it. The data paper should contain references to any research papers associated with the dataset and when referring to the data behind a study, a research paper should reference any existing data paper for further details.

JOPHD accepts any kind of public health data, including for example: epidemiology, ecology, environmental, and genotype data, geographic information system (GIS) data and maps, image and video data, quantitative and qualitative survey data.

\section{References}

1. Journal of Open Public Health Data (JOPHD) [Internet]. London: Ubiquity Press Limited. [Accessed 15 Aug 2013]. Available from: http://openpublichealthdata.metajnl.com/

2. Creative Commons Zero licence (CCo) [Internet]. California: Creative Commons Corporation. [Accessed 15 Aug 2013]. Available from: http://creativecommons.org/about/cco 